\title{
ON SOME PROPERTIES OF SYMMETRICAL PERFECT SETS ${ }^{1}$
}

\author{
R. SALEM
}

This paper deals with some properties of symmetrical perfect sets, in view of applications: (i) to the problem of multiplicity of trigonometrical series; (ii) to the study of Fourier-Stieltjes coefficients of singular monotone functions; (iii) to the problem of absolute convergence of trigonometrical series.

1. Sets of multiplicity of trigonometrical series. We shall consider, throughout the paper, symmetrical perfect sets, that is, sets which are obtained, in the closed interval $(0,2 \pi)$ by the following process. We divide the interval in three parts of lengths proportional to $\xi_{1}, 1-2 \xi_{1}$, $\xi_{1}$, and we remove the central open interval. In the second operation we divide each one of the two intervals left in three parts proportional to $\xi_{2}, 1-2 \xi_{2}, \xi_{2}$, and we remove the two central open intervals, and so on infinitely, the sequence $\left\{\xi_{p}\right\}$ being such that $0<\xi_{p} \leqq \frac{1}{2} .{ }^{2}$ After $p$ operations, we have thus removed $2^{p}-1$ intervals which we shall denote by $\delta_{p k}\left(k=1,2, \cdots, 2^{p}-1\right)$ and $2^{p}$ intervals are left, which we shall denote by $\eta_{p k}\left(k=1,2, \cdots, 2^{p}\right)$. Each interval $\eta_{p k}$ is of length equal to $\eta_{p}=2 \pi \cdot \xi_{1} \xi_{2} \cdots \xi_{p}$. Let $E_{p}$ be the set constituted by the $2^{p}$ intervals $\eta_{p k}$ and let $E(p)$ be its measure. We have

$$
E(p)=2^{p} \eta_{p}=2 \pi \cdot 2^{p} \xi_{1} \xi_{2} \cdots \xi_{p} .
$$

$E(p)$ is a non-increasing function of $p$ and the measure of the perfect set $P$ obtained by the above-described process is $\lim E(p)$ for $p=\infty$. We shall only consider, throughout the paper, sets for which this limit is equal to zero.

We shall now construct a monotone continuous function $F(x)$ constant in every interval contiguous to $P$ but increasing from one interval to another, by the following well known process. ${ }^{3}$ For every $p$ let $F_{p}$ be a non-decreasing continuous function defined by the following conditions: $F_{p}(0)=0, F_{p}(2 \pi)=1$,

$$
F_{p}(x)=\frac{k}{2^{p}} \text { in } \delta_{p k}, \quad k=1,2, \cdots, 2^{p}-1,
$$

1 Presented to the Society, May 3, 1941.

2 If $\xi_{p}=\frac{1}{2}$ no interval is removed in the $p$ th operation but the intervals left after the $(p-1)$ th operation are subdivided in two equal parts, and we deal in the $(p+1)$ th operation, with the intervals thus subdivided.

${ }^{3}$ See Menchoff [1]; Zygmund [1, p. 294]. 
and $F_{p}(x)$ linear in each $\eta_{p k}$. The consideration, in an interval $\eta_{p k}$, of the straight line representing $F_{p}$ and of the broken line representing $F_{p+1}$ shows immediately that

$$
\left|F_{p}-F_{p+1}\right| \leqq \frac{1}{2^{p+1}} \frac{\frac{1}{2} \eta_{p}-\eta_{p+1}}{\frac{1}{2} \eta_{p}}
$$

that is to say, by (1)

$$
\left|F_{p}-F_{p+1}\right| \leqq \frac{1}{2^{p+1}}\left[1-\frac{E(p+1)}{E(p)}\right] .
$$

Thus $F_{p}$ converges uniformly to a continuous function $F$ which is non-decreasing, is constant in every interval contiguous to $P$ but increasing from one interval to another, and we have $F(0)=0, F(2 \pi)=1$.

Let us consider now the Fourier-Stieltjes coefficients of $d F$. We have

$$
c_{n}=\int_{0}^{2 \pi} e^{n i x} d F=\int_{0}^{2 \pi} e^{n i x} d\left(F-F_{p}\right)+\int_{0}^{2 \pi} e^{n i x} d F_{p} .
$$

Denoting by $A$ and $B$ the two last integrals, and integrating $A$ by parts, we have, as $F-F_{p}$ vanishes for $x=0$ and $x=2 \pi$,

$$
|A| \leqq n \int_{0}^{2 \pi}\left|F-F_{p}\right| d x .
$$

Hence

$$
|A| \leqq n \int_{0}^{2 \pi}\left|F_{p}-F_{p+1}\right| d x+n \int_{0}^{2 \pi}\left|F_{p+1}-F_{p+2}\right| d x+\cdots .
$$

But

$$
\int_{0}^{2 \pi}\left|F_{p}-F_{p+1}\right| d x \leqq E(p) \cdot \max \left|F_{p}-F_{p+1}\right|,
$$

since $F_{p}=F_{p+1}$ when $x$ does not belong to $E_{p}$. Hence, by (2)

$$
\int_{0}^{2 \pi}\left|F_{p}-F_{p+1}\right| d x \leqq \frac{1}{2^{p+1}}[E(p)-E(p+1)] .
$$

Let us suppose now that $E(p)$ is a convex function; then $E(p)-E(p+1)$ is decreasing and we have

$$
|A| \leqq n[E(p)-E(p+1)] \sum_{\nu=p}^{\infty} \frac{1}{2^{p+1}}=\frac{n}{2^{p}}[E(p)-E(p+1)] .
$$

Considering now the integral $B$ we have 


$$
\int_{\eta_{p k}} e^{n i x} d F_{p}=\frac{1}{2^{p} \eta_{p}} \int_{\eta_{p k}} e^{n i x} d x=\frac{1}{E(p)} \int_{\eta_{p k}} e^{n i x} d x
$$

Hence

$$
|B| \leqq \frac{2^{p}}{E(p)} \cdot \frac{2}{n}
$$

and

$$
\left|c_{n}\right| \leqq \frac{n}{2^{p}}[E(p)-E(p+1)]+\frac{2^{p+1}}{n} \frac{1}{E(p)} .
$$

Up to here we have followed very closely the above quoted Menchoff's example, in extending it to general symmetrical sets. In Menchoff's example

$$
\xi_{p}=\frac{p}{2(p+1)}, \quad E(p)=\frac{1}{p+1} .
$$

Now the inequality (3) gives us in a very simple way a class of symmetrical perfect sets of measure zero which are sets of multiplicity. As is well known, in order that $P$ should be a set of multiplicity it is sufficient to have $c_{n}=o(1)$.

The integer $p$ being arbitrary, let us choose it satisfying the following condition:

$$
\begin{aligned}
& \frac{2^{p-1}}{(E(p-1))^{1 / 2}(E(p-1)}-E(p))^{1 / 2} \\
&<n \leqq \frac{2^{p}}{(E(p))^{1 / 2}(E(p)-E(p+1))^{1 / 2}}
\end{aligned}
$$

Thus, by (3)

$$
\left|c_{n}\right| \leqq\left(1-\frac{E(p+1)}{E(p)}\right)^{1 / 2}+4 \frac{E(p-1)}{E(p)}\left(1-\frac{E(p)}{E(p-1)}\right)^{1 / 2} .
$$

If $n$ increases infinitely, so does $p$ defined by (4); hence if we suppose $E(p+1) / E(p) \rightarrow 1$, we have $c_{n}=o(1)$.

We have thus obtained the following result: $:^{4}$ Each symmetrical perfect set of measure zero such that $E(p)$ is convex and $E(p+1) / E(p)$ tends to 1 is a set of multiplicity.

${ }^{4}$ A more general result is due to Nina Bary [1], but the proof is very complicated. 
2. The Fourier-Stieltjes coefficients of singular monotone functions. Let us now consider for every $p$ the characteristic function $f_{p}$ of the set $E_{p}$. We have

$$
\int_{0}^{2 \pi} e^{n i x} d F_{p}=\frac{1}{2^{p} \eta_{p}} \int_{E_{p}} e^{n i x} d x=\frac{1}{E(p)} \int_{0}^{2 \pi} f_{p}(x) e^{n i x} d x .
$$

Hence

$$
c_{n}=\int_{0}^{2 \pi} e^{n i x} d F=\frac{1}{E(p)} \int_{0}^{2 \pi} f_{p}(x) e^{n i x} d x+A,
$$

$A$ denoting, as above, the integral

$$
\int_{0}^{2 \pi} e^{n i x} d\left(F-F_{p}\right)
$$

We have seen that

$$
|A| \leqq n \sum_{\nu=p}^{\infty} \frac{1}{2^{\nu+1}}[E(\nu)-E(\nu+1)] .
$$

Here we shall make no hypothesis whatever about the behaviour of $E(p)-E(p+1)$. It is plain that in any case we have

Hence

$$
|A| \leqq \frac{n E(p)}{2^{p+1}} \text {. }
$$

$$
E^{2}(p)\left|c_{n}\right|^{2} \leqq 2\left|\int_{0}^{2 \pi} f_{p}(x) e^{n i x} d x\right|^{2}+2\left(\frac{n E^{2}(p)}{2^{p+1}}\right)^{2} .
$$

The integer $p$ is arbitrary. We can associate to every $n$ an integer $p=p(n)$ non-decreasing, tending to infinity with $n$, and sum the inequalities thus obtained from $n=1$ to $n=\infty$ provided that the sum of the right-hand side terms be convergent.

Let us observe that the sets $E_{p}$ are such that $E_{p+1} \subset E_{p}$ that is to say $f_{p+1} \leqq f_{p}$. Now it is known that in this case the sum

$$
S=\sum_{1}^{\infty}\left|\int_{0}^{2 \pi} f_{p}(x) e^{n i x} d x\right|^{2},
$$

where $p$ takes the same value for all $n$ such that $n_{p} \leqq n<n_{p+1}$, converges if $n_{p+1} / n_{p}>\lambda>1$; moreover $S<C(\lambda), C$ being a constant depending only on $\lambda$. This is a consequence of the well known theorem stating that if $S_{n}(x)$ is the $n$th sum of the Fourier series of a function $\phi$ of integrable square, then 


$$
\int_{0}^{2 \pi} \sup _{p}\left|S_{n_{p}}(x)\right| d x \leqq K(\lambda)\left[\int_{0}^{2 \pi} \phi^{2} d x\right]^{1 / 2}
$$

where $K(\lambda)$ depends on $\lambda$ only. ${ }^{5}$

Hence if we take, for example $p=[a \log n]$ ( $[m]$ denoting the integral part of $m$ ), $p$ will take the same value for $n_{p} \leqq n<n_{p+1}, n_{p}$ being given by

$$
a \log \left(n_{p}-1\right)<p \leqq a \log n_{p}
$$

Hence

$$
a \log n_{p+1}-a \log \left(n_{p}-1\right)>1
$$

that is to say,

$$
\frac{n_{p+1}}{n_{p}}>\lambda>1
$$

$\lambda$ depending on $a$ only.

On the other hand, we will have

$$
\frac{n}{2^{p+1}}<\frac{n}{2^{a \log n}}
$$

and it is easily seen that the series $\sum\left(n / 2^{p+1}\right)^{2}$ will converge if $a>3 /(2 \log 2)$.

Hence, returning to inequality (5) and writing $E[a \log n]$ instead of $E([a \log n])$, we get the following result:

The series

$$
T=\sum_{1}^{\infty} E^{2}[a \log n] \cdot\left|c_{n}\right|^{2}
$$

is convergent for $a>3 /(2 \log 2)$. Moreover $T<C(a), C$ being a constant depending on a only.

${ }^{5}$ See Zygmund [1, p. 252]; and for the equivalence of the two statements, Salem $[1,2]$. The proof is briefly as follows: $p=p(x)$ being any function of $x$, we have

$$
\left|\int_{0}^{2 \pi} S_{n_{p(x)}}(x) d x\right| \leqq K(\lambda)\left[\int_{0}^{2 \pi} \phi^{2} d x\right]^{1 / 2},
$$

and this can be written if $\phi(x) \sim \sum c_{n} e^{n i x}$,

$$
\left|\sum c_{n} \int_{0}^{2 \pi} f_{p} e^{n i x} d x\right| \leqq K_{1}(\lambda)\left(\sum\left|c_{n}\right|^{2}\right)^{1 / 2},
$$

where the $f_{p}$ are characteristic functions of sets, corresponding to $p(x)$, such that $f_{p+1} \leqq f_{p}$ and such that $f_{p}$ is the same for all $n$ for which $\left|n_{p}\right| \leqq|n|<\left|n_{p+1}\right|$. Since this holds good for any sequence $\left\{c_{n}\right\}$ such that $\sum\left|c_{n}\right|^{2}<\infty$, the statement about the sum $S$ follows immediately. 
The interest of this theorem lies in the fact that we can construct our perfect set $P$ of measure zero such that $E(p)$ tends to zero as slowly as we please. Hence, while the function $F(x)$ 's being singular (that is, constant in every interval contiguous to $P$ ) makes the series $\sum\left|c_{n}\right|^{2}$ necessarily divergent, the divergence of this series can be as slow as we please, and the rapidity of the divergence is connected in a very simple way with the function $E(p)$.

We refer the reader to other results which have been obtained previously in this field. See Wiener and Wintner [1, 2] and Schaeffer [1].

3. Absolute convergence of trigonometrical series. The set $P$ is said to be of the type $N$ if there exists a trigonometrical series $\sum \rho_{n} \cos \left(n x-\alpha_{n}\right)$ with $\rho_{n} \geqq 0, \sum \rho_{n}=\infty$, such that $\sum \rho_{n}\left|\cos \left(n x-\alpha_{n}\right)\right|$ $<\infty$ for every $x$ belonging to $P$. It has been proved ${ }^{6}$ that if $P$ is of the type $N$ we have

$$
\lim \sup \int_{0}^{2 \pi} \cos 2 n x d F=1
$$

If we split the sequence of integers $\{n\}$ in two complementary sequences $\left\{m_{k}\right\},\left\{n_{k}\right\}$ such that for every $k$ :

$$
\int_{0}^{2 \pi} \cos 2 m_{k} x d F \leqq 1-\epsilon, \quad \int_{0}^{2 \pi} \cos 2 n_{k} x d F>1-\epsilon,
$$

$\boldsymbol{\epsilon}$ being any fixed positive number $<1$, the proof of the theorem just stated shows that no series $\sum r_{k} \cos \left(m_{k} x-\alpha_{k}\right)$ can converge absolutely in $P$ unless $\sum r_{k}<\infty$. Hence, if the set $P$ is of the type $N$ every trigonometrical series absolutely convergent in $P$ but not everywhere can be broken into two complementary series: the first one;

$$
\sum r_{k} \cos \left(m_{k} x-\alpha_{k}\right) \quad \text { with } \sum r_{k}<\infty,
$$

which is a parasitic series convergent absolutely everywhere; and the second one, $\sum \rho_{k} \cos \left(n_{k} x-\beta_{k}\right)$, which converges absolutely in $P$, with $\sum \rho_{k}=\infty$.

Now, the question arises of what can be the frequency of the integers $\left\{n_{k}\right\}$. We can draw some conclusions from the theorem proved in $\$ 2$. We have, by (6), by a suitable choice of $\epsilon,\left|c_{2 n_{k}}\right|>\frac{1}{2}$. Hence

$$
\sum_{1}^{\infty} E^{2}\left[a \log 2 n_{k}\right]<\infty .
$$

\footnotetext{
${ }^{6}$ See Salem [3, p. 323].
} 
Roughly speaking, the more slowly $E(p)$ decreases, the more sparse are the integers $\left\{n_{k}\right\}$. More precisely if between $p$ and $q$ there are $\nu(p, q)$ integers of the sequence $\left\{n_{k}\right\}$ we have

$$
\nu \cdot E^{3}[a \log 2 q]<\sum_{p<n_{k}<q} E^{3}\left[a \log 2 n_{k}\right]<E[a \log 2 p] \cdot C(a) ;
$$

hence

$$
\nu<C(a) \cdot \frac{E[a \log 2 p]}{E^{3}[a \log 2 q]} .
$$

It has been shown ${ }^{7}$ that the perfect set $P$ for which all the $\xi_{p}$ are equal to $\frac{1}{2}$, except for a sequence $\left\{i_{j}\right\}$ for which $\xi_{i_{j}}=1 / 2 j$, is of the type $N$. By taking the sequence $\left\{i_{j}\right\}$ very sparse, we can plainly obtain a set of the type $N$ with $E(p)$ decreasing as slowly as we please. Hence, the inequality (7) shows that there exist sets of the type $N$ for which $\nu[p, \phi(p)]<\omega(p), \phi(p)$ increasing as rapidly, and $\omega(p)$ as slowly as we please.

This result throws some light on the fact that the sum of two sets $P, P^{\prime}$, both of the type $N$, may be not of the type $N$, a result which is due to Marcinkiewicz. ${ }^{8}$ If the sets $P, P^{\prime}$ are both of the type $N$, then, $F^{\prime}$ being the corresponding function for the set $P^{\prime}$, there exist two sequences of integers $\left\{n_{k}\right\},\left\{n_{k}^{\prime}\right\}$ such that

$$
\lim \int_{0}^{2 \pi} \sin ^{2} n_{k} x d F=0, \quad \lim \int_{0}^{2 \pi} \sin ^{2} n_{k}^{\prime} x d F^{\prime}=0
$$

by the theorem stated at the beginning of $\$ 3$. But it can be shown exactly in the same way that if $P+P^{\prime}$ is of the type $N$, we must have

$$
\lim \inf \left[\int_{0}^{2 \pi} \sin ^{2} n x d F+\int_{0}^{2 \pi} \sin ^{2} n x d F^{\prime}\right]=0 ;
$$

that is to say, there must exist a common sequence $\left\{m_{k}\right\}$ such that

$$
\lim \int_{0}^{2 \pi} \sin ^{2} m_{k} x d F=0, \quad \lim \int_{0}^{2 \pi} \sin ^{2} m_{k} x d F^{\prime}=0 .
$$

Now the sequences $\left\{n_{k}\right\},\left\{n_{k}^{\prime}\right\}$ satisfying (8) can be, as we have seen, both very sparse, hence the existence of a common sequence $\left\{n_{k}\right\}$ satisfying (9) may be impossible.

The question of what happens if the second set $P^{\prime}$ is reduced to a

${ }^{7}$ See Salem [3, p. 329].

${ }^{8}$ Marcinkiewicz [1]. 
single point has been mentioned to me by Dr. Marcinkiewicz and Professor Zygmund. We will show here that the sum of any set of the type $N$ (not necessarily a perfect set) and of a single point is also of the type $N$.

It has been proved ${ }^{9}$ that by a suitable translation of the given set $E$ we can suppose that the series converging absolutely in $E$ is a sine series. Let us suppose, then, that for every $x$ belonging to $E$ we have

$$
\sum_{1}^{\infty} \rho_{n}|\sin n x|<\infty, \quad \rho_{n} \geqq 0, \sum \rho_{n}=\infty .
$$

We can always find a positive function $\omega(n)$ increasing infinitely with $n$, such that

$$
\sum_{1}^{\infty} \frac{\rho_{n}}{\omega(n)}=\infty, \quad \sum_{1}^{\infty} \frac{\rho_{n}}{\omega^{2}(n)}<\infty .
$$

(We can take, for example, $\omega(n)=\sum_{1}^{n} \rho_{n}$.) Let $Q$ be a point of abscissa $x_{0}$, not belonging to $E$. By Dirichlet's theorem, for every $n$ we can find an integer $p_{n}$ such that

$$
1 \leqq p_{n} \leqq \omega(n), \quad\left|p_{n}\left(n \frac{x_{0}}{\pi}\right)-h_{n}\right|<\frac{1}{[\omega(n)]},
$$

$p_{n}$ being an integer and $(z)=z-[z]$ denoting the fractional part of $z$. Thus

$$
\left|p_{n} n \frac{x_{0}}{\pi}-p_{n}\left[n \frac{x_{0}}{\pi}\right]-h_{n}\right|<\frac{1}{[\omega(n)]} ;
$$

that is to say,

$$
\left|p_{n} n x_{0}-k_{n} \pi\right|<\frac{4}{\omega(n)},
$$

$k_{n}$ being an integer. Thus

$$
\left|\sin p_{n} n x_{0}\right|<\frac{4}{\omega(n)}
$$

for every $n$. Hence

$$
\sum_{1}^{\infty} \frac{\rho_{n}}{\omega(n)}\left|\sin p_{n} n x_{0}\right|<4 \sum_{1}^{\infty} \frac{\rho_{n}}{\omega^{2}(n)}<\infty .
$$

${ }^{9}$ See Salem [3, p. 319]. 
On the other hand, applying the well known theorem: $|\sin m t|$ $\leqq m|\sin t|$ for $m$ a positive integer, we have, by (10), if $x$ belongs to $E$ :

$$
\begin{aligned}
\sum_{1}^{\infty} \frac{\rho_{n}}{\omega(n)}\left|\sin p_{n} n x\right| & \leqq \sum_{1}^{\infty} \frac{p_{n}}{\omega(n)} \rho_{n}|\sin n x| \\
& \leqq \sum_{1}^{\infty} \rho_{n}|\sin n x|<\infty .
\end{aligned}
$$

Hence, by (11) and (12) the series $\sum_{1}^{\infty}\left[\rho_{n} / \omega(n)\right] \sin n x$ converges absolutely in $E+Q$. But $\sum_{1}^{\infty} \rho_{n} / \omega(n)=\infty$; hence $E+Q$ is of the type $N$.

This leads immediately to the following result: The sum of any set of the type $N$ and of any finite set is also of the type $N$.

\section{BIBLIOGRAPHY}

N. BARY

1. Sur l'unicitê du développement trigonométrique, Fundamenta Mathematicae, vol. 9 (1927), pp. 62-118.

J. MARCINKIEWICZ

1. Quelques théorèmes sur les séries et les fonctions, Travaux de la Société des Sciences et des Lettres de Wilno, Classe des Sciences Mathématiques et Naturelles, vol. 12 (1937).

D. MENCHOFF

1. Sur l'unicitê $d u$ développement trigonométrique, Comptes Rendus de l'Académie R. SALEM des Sciences, Paris, vol. 163 (1916), pp. 433-436.

1. Sur une méthode de sommation, valable presque partout, pour les séries de Fourier de fonctions continues, Comptes Rendus de l'Académie des Sciences, Paris, vol. 205 (1937), p. 14.

2. Essais sur les Séries Trigonométriques, Paris, Hermann, 1940.

3. The absolute convergence of trigonometrical series, Duke Mathematical Journal, vol. 8 (1941), pp. 317-334.

A. C. Schaeffer

1. The Fourier-Stieltjes coefficients of a function of bounded variation, American Journal of Mathematics, vol. 61 (1939), pp. 934-940.

N. WIENER AND A. WINTNER

1. Fourier-Stieltjes transforms and singular convolutions, American Journal of Mathematics, vol. 60 (1938), pp. 513-532 and Bibliography.

A. ZYGMUND

1. Trigonometrical Series, Warsaw, 1935.

Montreal, Quebec, CANada 